\title{
Médiévales
}

Langues, Textes, Histoire

52 | printemps 2007

Le livre de science, du copiste à l'imprimeur

\section{Dominique Rigaux, Le Christ du dimanche, histoire d'une image médiévale}

Paris, l'Harmattan, 2005, 502 p.

Dominique Donadieu-Rigaut

\section{CpenEdition}

\section{Journals}

Édition électronique

URL : https://journals.openedition.org/medievales/2753

DOI : 10.4000/medievales.2753

ISSN : 1777-5892

\section{Éditeur}

Presses universitaires de Vincennes

Édition imprimée

Date de publication : 1 juin 2007

Pagination : 199-201

ISBN : 978-2-84292-202-3

ISSN : 0751-2708

Référence électronique

Dominique Donadieu-Rigaut, " Dominique Rigaux, Le Christ du dimanche, histoire d'une image

médiévale », Médiévales [En ligne], 52 | printemps 2007, mis en ligne le 11 février 2008, consulté le 24 avril 2022. URL : http://journals.openedition.org/medievales/2753 ; DOI : https://doi.org/10.4000/ medievales. 2753 
de Jean Favier, ou celles plus récentes de Jean-François Lassalmonie et de Guillaume Leyte notamment, la vigueur de ces conclusions suffit à souligner l'importance du travail de L. Scordia dans la perspective d'une histoire culturelle de l'État médiéval.

Julie Mayade-Claustre

Dominique Rigaux, Le Christ du dimanche, histoire d'une image médiévale, Paris, l'Harmattan, 2005, 502 p.

Avec cet ouvrage, Dominique Rigaux met en évidence le rôle des images dans l'instauration, ou plutôt la réaffirmation d'une pratique sociale, celle du repos prôné par l'Église le dimanche et les jours fériés. La question posée est donc large puisqu'elle engage la conception du temps hebdomadaire dans le monde chrétien, les rapports travail/temps libre, et surtout la mise au point d'une représentation très singulière, «le Christ du dimanche », qui n'avait guère attiré l'attention des historiens de l'art jusqu'ici.

L'auteur commence par rappeler les étapes de la distinction de ce jour singulier qu'est le dimanche, pour les Chrétiens, au sein de la semaine. Son émergence constitue un phénomène hautement identitaire : il permet non seulement à la communauté chrétienne de se positionner par rapport aux Juifs qui prohibent, eux, toute activité lors du sabbat, mais il révèle également à quel point la communauté chrétienne s'est construite de l'intérieur autour d'une pratique sacramentelle, en l'occurrence l'Eucharistie. Car si, dès l'époque patristique, l'interruption du travail est préconisée le « jour du Seigneur» (Dominica dies), c'est moins pour se reposer du labeur des six jours précédents que pour permettre aux fidèles de se rassembler afin de prendre part, collectivement, au rituel de la messe. D'ailleurs, la durée de ce repos dominical fait débat, certains, comme saint Augustin, pensant qu'une journée entière d'oisiveté risque de pousser les chrétiens au péché («Mieux vaut labourer que danser »). Les partisans d'un simple moment de loisir dans la journée, juste nécessaire pour se rendre à l'église et participer au culte, font entendre leur voix.

Toujours est-il qu'à partir du vI ${ }^{\mathrm{e}}$ siècle, l'hagiographie, à la suite des conciles mérovingiens, vient à la rescousse de ce jour pas comme les autres. La vie de saint Martin de Tours, notamment, évoque les punitions divines réservées à ceux qui osent enfreindre l'interdit: la main du forgeron reste collée à la clé qu'il est en train de fabriquer, celle du laboureur se fige sur la charrue, celle de la femme prenant soin de sa chevelure se pétrifie sur le peigne. Dans tous les cas, la paralysie, antithèse de l'activité, frappe la part du corps engagée dans l'action en la scellant à l'objet ou à l'outil manipulé.

Le spectre des activités prohibées le dimanche est donc large, englobant non seulement le travail productif (agricole, artisanal ou commercial), les tâches domestiques (laver le linge...), les relations sexuelles, les déplacements de longue distance, mais aussi tout acte visant à soigner ou embellir le corps, notamment féminin. Charlemagne, en 789, dans son Admonitio generalis, établit une liste complète de ces activités, en les classant soigneusement par rubrique. Même si des dispenses ont été régulièrement accordées par les évêques pour telle ou telle activité temporaire ou spécifique, cette liste ne fut jamais remise en cause au cours du Moyen Âge. Seuls les ordres Mendiants, soucieux d'encourager les œuvres de miséricorde, l'ont modulée, en précisant qu'un travail bénévole effectué le dimanche par charité ne pouvait être condamnable et donc condamné.

À partir du milieu du XIV $\mathrm{X}^{\mathrm{e}}$ siècle apparaissent des images donnant à voir, principalement sur les murs (intérieurs ou extérieurs) des églises, un Christ debout, la plupart 
du temps nu, grandeur nature, agressé par les symboles des activités défendues. Dominique Rigaux en a établi un corpus (presque 100 occurrences) entièrement publié en deuxième partie de l'ouvrage. Le regroupement de tous ces documents en un seul livre permet au lecteur de prendre conscience à la fois de la grande cohérence du corpus et des subtiles variations qui font de chaque image un cas unique. En s'appuyant sur l'étude des écarts - minimes ou plus importants - entre les représentations, l'auteur élabore une typologie des «Christ du dimanche ». Certains documents montrent le Fils de Dieu littéralement agressé par des outils piquants ou tranchants (ciseaux, pinces, faucilles, vrilles, socs de charrue...) plantés dans son corps, produisant ainsi une multitude de plaies sanguinolentes. D'autres images mettent plutôt en scène une «oppression christique », l'Incarné étant entouré d'outils pointés vers lui formant une sorte de mandorle menaçante. D'autres encore présentent un Christ souffrant, en pied, au milieu d'un bric-à-brac d'outils emplissant toute la surface de la peinture murale. Parfois, quelques gouttes de sang viennent «orner » les instruments manipulés au mauvais moment. Enfin, certaines représentations élaborent de véritables systèmes logiques en matérialisant, par des fils de sang ou bien des flèches, les liens de cause à effet entre la souffrance corporelle du Christ et les activités indûment effectuées les jours chômés. Il peut arriver que de petites scènes de travail (et non simplement des outils décontextualisés), symbolisent les activités humaines : on peut alors voir sur le dos des Chrétiens affairés de minuscules démons, tout noirs, les poussant à enfreindre la loi du calendrier.

Outre le mérite d'attirer l'attention sur une représentation encore peu connue, cette étude présente un intérêt méthodologique majeur: celui de démontrer qu'un thème iconographique donné ne se constitue pas en lui-même mais qu'il se situe toujours à la confluence d'autres thèmes. À cet égard, l'auteur replace son image au cœur d'un réseau de représentations : l'Homme de douleur, les Arma Christi, la messe de saint Grégoire, le pressoir mystique, mais les représentations des métiers et des travaux des champs ne sont pas non plus étrangères au «Christ du dimanche ». Ainsi, il apparaît clairement que cette image, au premier abord déroutante, est partie prenante d'un ensemble de croyances et de phénomènes culturels qui se manifestent à la fin du Moyen Âge autour du culte du Saint-Sang, de l'Eucharistie, des processions de la Fête-Dieu, ou encore de la méditation sur les cinq plaies. Dans ce contexte, travailler le dimanche, c'est renouveler la Passion du Christ, se faire le bourreau de son corps au mépris du sang déjà versé sur la croix. Visuellement, les cinq blessures christiques se trouvent ravivées par le péché (elles saignent alors abondamment), à moins que les activités des hommes n'ajoutent encore d'autres blessures au corps du Christ faisant de lui un martyr aux allures de saint Sébastien. Aussi, le sang rédempteur se transforme en sang accusateur : si le précieux liquide rachète toutes les fautes ou presque, les Chrétiens doivent faire en sorte de ne pas le faire couler à nouveau et inutilement. À cet égard, l'auteur souligne que le «Christ du dimanche » est moins à entendre comme une illustration directe du décalogue que comme un «contremodèle, un condensé de ce qu'il ne faut pas faire ».

Dominique Rigaux intègre à sa série quelques «images-limites » qui reprennent le même thème en substituant au Christ un personnage féminin, voire androgyne. Ces représentations énigmatiques, que certains ont analysées comme renvoyant à une Vierge protectrice des métiers, seraient en fait une sanctification du dimanche passant par la personnification féminine de ce jour férié. D'après l'auteur, cette «dame du dimanche », surtout présente dans les zones italophones, est la preuve de l'emprise du genre des mots sur les images. En effet, en italien, le dimanche est un mot féminin 
(la Domenica). Cette question ouvre donc des pistes nouvelles dans l'étude des rapports complexes entre la langue et les images, mais aussi dans celle de l'élaboration iconographique des types sexués.

Toutes ces représentations, qu'elles soient féminines, androgynes ou masculines, ont été commanditées par des communautés villageoises et réalisées par de petits maîtres d'origine paysanne appartenant à des ateliers itinérants. L'auteur, familière des peintures murales de l'arc alpin, retrouve ici son terrain de prédilection, puisqu'une bonne part de son corpus relève des vallées alpines, même si certaines fresques ont vu le jour en Angleterre, d'autres en Italie centrale (voire dans les environs de Naples). La Péninsule ibérique et la France (hormis les actuelles AlpesMaritimes) semblent avoir ignoré «le Christ du dimanche». Plusieurs cartes en couleurs, très claires, témoignent de l'extension de ce thème iconographique. C'est à ce niveau également que le livre donne à réfléchir. En effet, quelle(s) échelle(s) d'approche adopter pour analyser un tel phénomène iconographique intrinsèquement lié aux églises rurales, notamment montagnardes, mais concernant in fine un espace très vaste (des Grisons à la Slovénie) dépassant à l'évidence les intérêts locaux ? Probablement des échelles combinées, rendant compte à la fois de l'enracinement paroissial de ces images et de la structuration d'un temps chrétien qui engage l'ensemble de la Chrétienté. Ou plutôt des temps chrétiens. Car si le calendrier romain vise à unifier l'espace en imposant des rythmes communs à l'ensemble des fidèles, chaque diocèse conserve en matière de fêtes sanctorales (et donc aussi de temporalité) une part de flexibilité, de spécificité. L'emplacement même de ces peintures murales, sur les murs des églises paroissiales, résonne ainsi comme une invitation à s'insérer dans le calendrier chrétien « localement» en investissant collectivement, le jour du Seigneur, le lieu dévolu à l'Eucharistie : respecter et honorer le dimanche le corps et le sang $\mathrm{du}$ Christ, c'est donc aussi s'incorporer aux dimensions spatiales et temporelles de la Chrétienté, même et surtout lorsque l'on vit dans un village reculé de montagne.

Dominique Donadieu-Rigaut

Antoine Franzini, La Corse du XV siècle. Politique et société, 1433-1483, Ajaccio, Alain Piazzola, 2005, 750 p.

La Corse $\mathrm{du} \mathrm{XV}^{e}$ siècle est tiré d'une thèse de doctorat soutenue à l'université de Corse en avril 2003 par Antoine Franzini, que l'on connaît déjà comme l'auteur d'un bon nombre de travaux sur l'histoire de la Corse à l'époque médiévale. Le livre entend donner de la Corse au $\mathrm{Xv}^{\mathrm{e}}$ siècle une image riche et précise, comme le montrent son titre ambitieux et son volume imposant. Et le pari est tenu : impossible de résumer ici ces presque sept cents pages de texte, suivies d'annexes solides (publication de quelques pièces, comme les lettres d'Antonio Ivani, bibliographies et index).

«Politique et société » annoncées par le sous-titre (mais, disons-le d'emblée, il est aussi parfois question d'économie, de religion et de culture), étudiées à l'échelle régionale et sur une durée moyenne : on n'est pas loin, avec cet objet et cette méthode finalement assez classiques, de ces grandes thèses d'État qui furent longtemps la marque de fabrique de l'historiographie nationale. L'île souffre d'un net déficit bibliographique qui, de façon assez extraordinaire, s'est toujours fait sentir - que l'on se réfère à la citation liminaire du plus célèbre des chroniqueurs corses, Giovanni Della Grossa : en plein $\mathrm{XV}^{\mathrm{e}}$ siècle, il écrivait que «la pauvre Corse » n'avait pas encore eu d'hommes «qui aient noté pour [son] temps de manière intelligible les événements nouveaux qui se sont produits ». C'est ce manque qu'entend combler l'auteur, conscient des nombreux lieux communs et affabulations qu'a entraînés le 